\title{
Immunoisolation of stem cells by simultaneous encapsulation and PEGylation
}

\author{
Roya Ramezanzadeh Andevari ${ }^{1}$ - Sameereh Hashemi-Najafabadi ${ }^{1}$. Fatemeh Bagheri ${ }^{2}$
}

Received: 18 July 2017 / Accepted: 6 February 2018 / Published online: 19 February 2018

(C) The Author(s) 2018. This article is an open access publication

\begin{abstract}
Today, cell therapy is known as an important tool in the treatment of chronic diseases where cells lose their normal function. Immunoisolation systems using microencapsulation or PEGylation have been developed to evade the problem of rejection by the immune system. The aim of the present study was to investigate a combination of microencapsulation and PEGylation methods in coating mouse embryonic stem cells (mESCs) to determine its effect in reducing the host's immune response. Therefore, methoxy polyethylene glycol (mPEG) binding on alginate-trimethyl chitosan (TMC) microcapsules was investigated using FTIR. Furthermore, survival of the microencapsulated mESCs was confirmed using AO/PI staining and MTT assays. In addition, the effect of mESCs co-cultured with foreign lymphocytes was evaluated. Overall, interleukin-2 (IL-2) secretions as a response of the immune system revealed that mESCs microencapsulation in alginate-TMC-PEG, reduced the immune system response. The results suggested that IL-2 secretion was reduced to $62 \%$ at seventh day.
\end{abstract}

Keywords Microencapsulation · PEGylation · Alginate $\cdot$ Embryonic stem cells (ESCs) · Trimethyl chitosan (TMC)

\section{Introduction}

Cell therapy is an effective method in transplantation medicine. It is a multidisciplinary field between immunology, biomaterials and regenerative medicine. The main objective of cell therapy is to replace the function of injured tissues (Hernández et al. 2010). Existing obstacles in obtaining optimum populations of specific cell lines for cell therapy limit the clinical cell transplantation. Stem cells, including pluripotent embryonic stem cells (ESCs), have high potential for cell therapy; subsequently to overcome the existing limitations of donor cells.

Microencapsulation is a process by which the biologically active materials are enclosed within micro-spherical and semi-permeable containers of 0.2-3.0 mm in diameter (Paredes Juarez et al. 2014; Al-Rammah 2014; Azadi et al. 2016). Alginate is an appealing material that has been widely used for cell encapsulation (Wang et al. 2009).

Sameereh Hashemi-Najafabadi

s.hashemi@modares.ac.ir

1 Department of Biomedical Engineering, Faculty of Chemical Engineering, Tarbiat Modares University, Tehran, Iran

2 Department of Biotechnology, Faculty of Chemical Engineering, Tarbiat Modares University, Tehran, Iran
Proliferation of the encapsulated stem cells (SCs) may depend on the polymer concentration and medium condition (Hashemi and Kalalinia 2015). For example, Wang et al. (2009) showed that ESCs encapsulation within 1\% (w/v) alginate gel provides optimal conditions for cell proliferation in comparison to a less or more alginate present. Also, they reported that a modified alginate-based 3D strategy supported proliferation and differentiation of the mouse ESCs (mESCs) into pancreatic insulin-producing cells (Wang et al. 2009; Chayosumrit et al. 2010).

Kim et al. modified the culture conditions to enhance the viability of encapsulated human embryonic stem cells (hESCs) in $1.1 \%$ calcium alginate. They showed significantly enhanced viability of the encapsulated hESCs (Kim et al. 2013). Moshaverinia et al. (2013) also developed a novel co-delivery system of RGD-coupled alginate hydrogel containing TGF-b1 for microencapsulation of dental mESCs.

The presence of the second layer in multilayered alginate microcapsules, typically using a polycation, such as poly-L-lysine (PLL) or chitosan, serves as the second barrier against the host immune system by decreasing the permeability of larger molecules (Tam et al. 2011).

PEGylation, covalent attachment of polyethylene glycol (PEG), is an alternative method for immunoisolation in cell 
therapy. The effect of PEGylation using activated methoxy PEGs (mPEGs) on the islets of Langerhans has been studied before, though single layer PEGylation cannot fully protect the cells from the host immune system. Nabavimanesh et al. (2015) developed a new design using simultaneous encapsulation and PEGylation to protect the islets.

In the present study, we investigated simultaneous encapsulation and PEGylation, to immunocamouflage ESCs as a source of cell for future differentiation applications. In this approach, trimethyl chitosan (TMC) was used as a polycation, and activated mPEG was attached on the surface of alginate-TMC microcapsules. Finally, the encapsulated cells viability and immunoprotection property of the microcapsules were investigated.

\section{Materials and methods}

\section{Materials}

High $G$ alginate, mPEG-succinimidyl valeric acid (mPEG-SVA) $(10 \mathrm{kDa})$ and acridine orange $(\mathrm{AO})$ were purchased from BDH (UK), Lysan Bio Inc and Merck (Darmstadt, Germany), respectively. TMC was prepared in Biomedical Engineering Department, Tarbiat Modares University. Dulbecco's modified eagle medium (DMEM), fetal bovine serum (FBS) and fetal calf serum (FCS) were obtained from Gibco (Carlsbad, CA, USA). RPMI-1640 medium, trypsin/EDTA, and penicillin/streptomycin antibiotics were prepared from Gibco. L-glutamine, $B$-mercaptoethanol and leukemia inhibitory factor were purchased from Invitrogen, Sigma and Chemicon, respectively. Mouse IL-2 kit was acquired from e-Bioscience (San Diego, CA, USA).

\section{Methods}

\section{Cell-free microcapsule preparation}

Alginate microcapsules were prepared based on Lim and Sun's method (Sun 1988), and has been explained in our previous work (Nabavimanesh et al. 2015). The alginate beads were transferred to TMC solution ( $1 \% \mathrm{w} / \mathrm{v}$ in saline) for $10 \mathrm{~min}$, and the prepared microcapsules were washed twice in saline. Finally, the obtained microcapsules were PEGylated using mPEG-SVA, according to our previous work (Nabavimanesh et al. 2015).

\section{Fourier transform infrared analysis (FTIR)}

The presence of TMC and mPEG on the alginate microcapsules was evaluated using FTIR spectroscopy (Perkin Elmer, $500-4000 \mathrm{~cm}^{-1}$ ).

\section{Cell culture}

Rat mesenchymal stem cells (rMSCs) were used as model cells in some early experiments due to some limitations in obtaining ESCs. Bone marrow was extracted from the femur of 8- to 10-week-old male Wistar rats, and was established in DMEM medium containing $15 \%$ FBS and $100 \mathrm{U} / \mathrm{mL}$ penicillin/streptomycin. The culture was then refreshed twice weekly. At this time, the cells were lifted by trypsin/EDTA and split into two $75 \mathrm{~cm}^{2}$ flasks containing fresh medium. In further successive subcultures, rMSCs population was increased.

mESCs were purchased from Royan Institute and maintained at undifferentiated state in gelatin-coated T-25 flasks in DMEM containing 10\% FCS, 2 mM L-glutamine, $1 \%$ penicillin/streptomycin (100 U/mL/0.1 mg/mL), $0.1 \%$ B-mercaptoethanol and $1000 \mathrm{U} / \mathrm{mL}$ leukemia inhibitory factor.

\section{Encapsulation of SCs}

The alginate solution was prepared by dissolving $2 \mathrm{~g}$ alginate in $100 \mathrm{~mL}$ of $\mathrm{Ca}^{2+}$ free DMEM. Adherent SCs were removed following trypsin incubation, and the cell number and viability were investigated using trypan blue exclusion (Wang et al. 2009). According to previously described method, mESCs at a density of $2 \times 10^{6}$ cells per mL of alginate solution were encapsulated in alginate-TMC-PEG. The entire process of encapsulation was performed under sterile conditions.

\section{Alginate beads solubilization and cell recovery}

To determine the average number of cells per capsule, a sample of the prepared capsules was incubated with a solution containing $50 \mathrm{mM}$ sodium citrate, $10 \mathrm{mM}$ glucose and $27 \mathrm{mM} \mathrm{NaCl}$ for $30 \mathrm{~min}$ at $37^{\circ} \mathrm{C}$. The suspension was centrifuged at $1200 \mathrm{rpm}$ for $6 \mathrm{~min}$, and the sodium citrate solution was aspirated. The pellet was then washed twice with PBS buffer, and re-suspended in DMEM medium for cell counting by trypan blue exclusion (Wang et al. 2009).

\section{rMSCs viability and proliferation}

Viability and proliferation of the encapsulated cells were investigated using MTT assay. MTT assay was performed after 1, 3, 5 and 7 days of culture with three replicates. A sample of $500 \mu \mathrm{L}$ was removed from each well and replaced with fresh medium. One hundred microliter of MTT solution ( $5 \mathrm{mg} / \mathrm{mL}$ in PBS) was added immediately to each sample. The microcapsules were incubated in MTT-containing medium for $4 \mathrm{~h}$ at $37^{\circ} \mathrm{C}$ under $5 \% \mathrm{CO}_{2}$. After incubation, the 
medium was removed completely, and $500 \mu \mathrm{L}$ of dimethyl sulfoxide (DMSO) was added into each sample to dissolve the formazan crystals. Absorbance of the obtained solution was measured using a microplate reader at $545 \mathrm{~nm}$.

\section{Lymphocytes isolation}

The lymphocytes were isolated from male C57B1/6 mice (12 weeks old, 25-30 g in weight), according to our previous work (Nabavimanesh et al. 2015).

\section{Studying the immunological reactions}

The immunological reactions were assessed by co-culturing approximately $10^{6}$ encapsulated or free mESCs with $5 \times 10^{5}$ lymphocytes in each well of a 24-well plate in $1 \mathrm{~mL}$ of DMEM/F12 medium under $5 \% \mathrm{CO}_{2}$ and $37{ }^{\circ} \mathrm{C}$ up to 7 days. IL-2 secretion by the lymphocytes co-cultured with ESCs encapsulated in alginate, alginate-TMC, and alginate-TMC-mPEG microcapsules, was assessed according to our previous work (Nabavimanesh et al. 2015).

\section{mESCs viability after co-culturing with lymphocytes}

mESCs viability was determined by staining with AO/PI. The stained encapsulated $\mathrm{mESCs}$ in alginate, alginate-TMC, and alginate-TMC-mPEG microcapsules, were assessed using fluorescent microscopy (IX71 OLYMPUS) 7 days after co-culturing with lymphocytes. The mESCs in microcapsules showing green are considered as viable cells.

\section{Results}

\section{FTIR analysis}

FTIR spectra obtained for alginate, alginate-TMC, and alginate-TMC-mPEG microcapsules are shown in Fig. 1. It shows that there are several differences between the spectra of alginate, alginate-TMC and alginate-TMC-mPEG microcapsules (in 1000-1200 cm $\mathrm{cm}^{-1}, 1300-1500 \mathrm{~cm}^{-1}$, $1500-1700 \mathrm{~cm}^{-1}$ and $3000-3500 \mathrm{~cm}^{-1}$ regions) that confirm the attachment of TMC and mPEG on the alginate and alginate-TMC microcapsules, respectively.

\section{Bead solubilization and cell recovery}

After solubilization of alginate microcapsules, the obtained cells were counted using trypan blue exclusion. rMSCs at a density of $1 \times 10^{6} \mathrm{cell} / \mathrm{mL}$ in alginate solution were encapsulated in three repetitions, and the obtained results showed a cell loading of about $237 \pm 7$ cells per capsule.

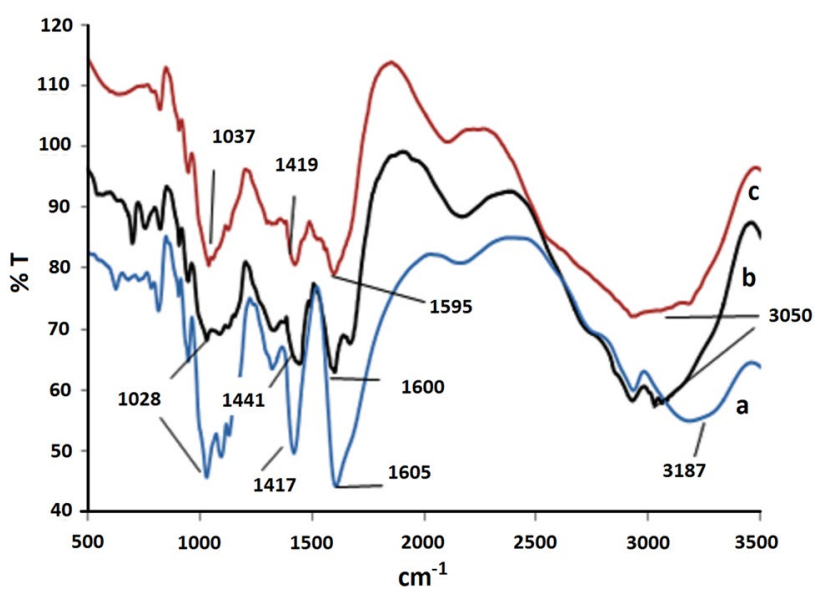

Fig. 1 FTIR spectra for alginate (a), alginate-TMC (b), and alginateTMC-mPEG (c) microcapsules

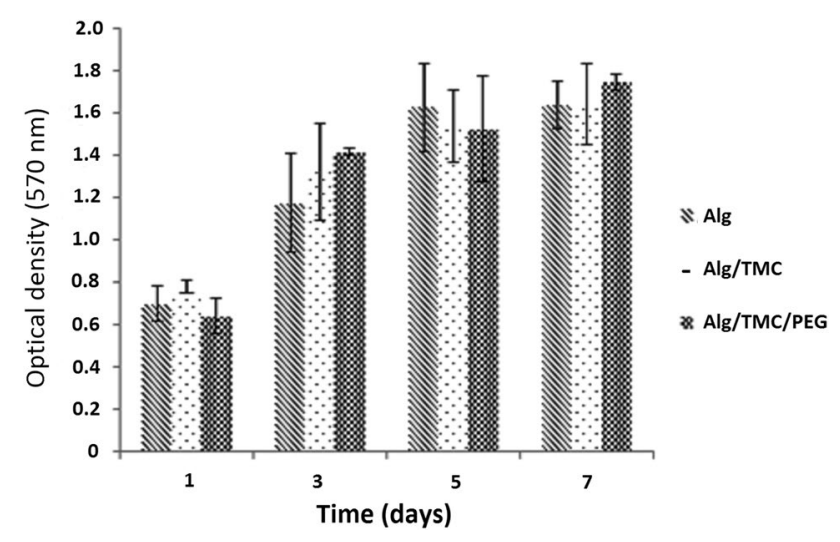

Fig. 2 MTT results for the cells encapsulated in alginate, alginateTMC, and alginate-TMC-PEG microcapsules

\section{Cells viability and proliferation}

Proliferation and viability of the encapsulated cells were measured using MTT assay. Proliferation of MSCs after 1, 3, 5 and 7 days of culture is shown in Fig. 2. MTT assay results indicated that three layers of alginate, TMC and mPEG are biocompatible for MSCs. As it can be seen, MSCs show their highest level of proliferation after 5 days.

\section{Immunological reactions against the mESCs}

Figure 3 shows IL-2 secretion from the lymphocytes against the mESCs in alginate, alginate-TMC and alginate-TMC-mPEG-SVA microcapsules. Every experiment was replicated twice. According to Fig. 3, IL-2 secretion increased overtime in all the samples.

The lymphocytes co-cultured with mESCs encapsulated in alginate showed less IL-2 secretion compared to one with 


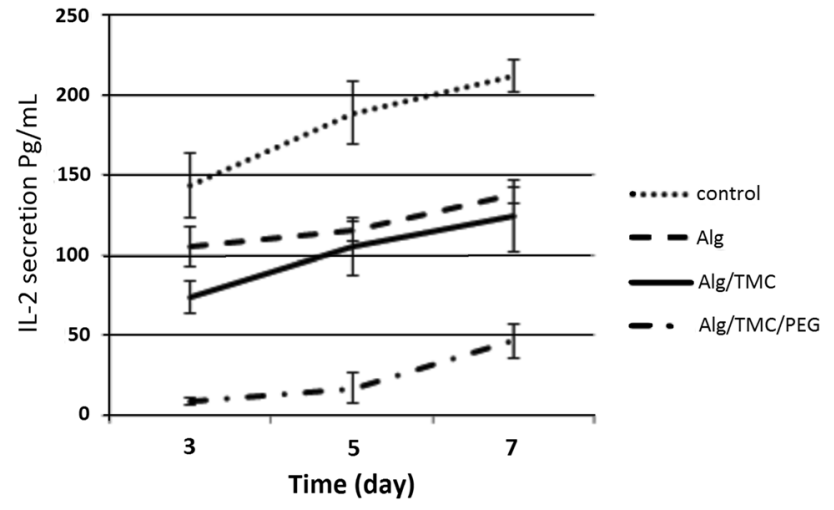

Fig. 3 Immunological response against the free and encapsulated mESCs based on IL-2 secretion from the lymphocytes, after 3, 5, and 7 days of co-culturing with free mESCs, and mESCs encapsulated in alginate, alginate-TMC and alginate-TMC-mPEG-SVA microcapsules, separately

free mESCs, due to the formation of an immunological barrier. On the 7 th day, this response decreased by $35 \%$ in comparison with free mESCs. TMC addition on the alginate microcapsules decreased IL-2 secretion against the mESCs in comparison with free mESCs and also, it was $9.4 \%$ less than the secretion against the mESCs in alginate microcapsules on the 7th day.

As shown in Fig. 3, grafting mPEG-SVA $(0.1 \%$ w/v) on the surface of alginate-TMC microcapsules also reduced the IL-2 secretion. Therefore, PEGylation prevents the immune cells and improves the immune protection. Moreover, the extent of IL-2 secretion against the mESCs in alginate-TMC-mPEG microcapsules over time was less than that for mESCs in microcapsules without mPEG. For mESCs in alginate-TMC-mPEG-SVA microcapsules; IL-2 secretion was reduced by $62 \%$ in comparison with the mESCs in alginate-TMC on the 7th day.

\section{mESCs viability after co-culturing with lymphocytes}

Viability of the free mESCs and encapsulated mESCs in alginate, alginate-TMC, and alginate-TMC-MPEG microcapsules was examined after 7 days of co-culturing with lymphocytes. Figure 4 demonstrates that $\mathrm{mESCs}$ were viable for 7 days after encapsulation.

\section{Discussion}

Encapsulation and PEGylation are two different methods of immunoisolation in cell transplantation. They have been used simultaneously for islets immunocamouflaging for the first time in our group (Nabavimanesh et al. 2015). The present study investigated simultaneous encapsulation and
PEGylation for entrapment of mESCs and we used ESCs to overcome the existing limitations in donor cells.

FTIR spectra of $\mathrm{C}-\mathrm{O}-\mathrm{C}$ bond at $1030 \mathrm{~cm}^{-1}$ for alginate, alginate-TMC, and alginate-TMC-PEG microcapsules are related to alginate (Coates 2000; Hu et al. 2013). The observed peak at $1441 \mathrm{~cm}^{-1}$ in alginate-TMC spectrum is related to $\mathrm{N}-\mathrm{CH}_{3}$ bond of amino group that indicates the presence of TMC layer. The angular deformation of $\mathrm{N}-\mathrm{H}$ bond of amino groups occurs at $1600 \mathrm{~cm}^{-1}$ (1500-1620 $\left.\mathrm{cm}^{-1}\right)$, though it is weaker due to $N$-methylation in TMC (Mourya and Inamdar 2009). Intensity of the peak at $1030 \mathrm{~cm}^{-1}$ increased in alginate-TMC-mPEG microcapsules due to grafting mPEG-SVA on alginate-TMC microcapsules. The observed peak at $1600 \mathrm{~cm}^{-1}$ is associated with the presence of amino I group (bending bond) (Sadeghi et al. 2004). The reduced peak intensity for alginate-TMC-mPEG microcapsules, in comparison with alginate-TMC, could be related to the bonding of $\mathrm{NH}_{3}{ }^{+}$groups of TMC to $\mathrm{COO}^{-}$groups of mPEG. Additionally, the observed peak at $3000-3500 \mathrm{~cm}^{-1}$ is related to hydroxyl and stretching amino groups. Figure 1 shows that addition of a layer to the microcapsules would result in a wider peak in this range. This clearly indicates the presence of TMC on alginate-TMC microcapsules and mPEG on alginate-TMC-mPEG microcapsules corresponding to their amino groups (Coates 2000; Trif and Socaciu 2007; Pavia et al. 2008).

MTT results suggested that encapsulation of the cells in alginate, alginate-TMC and alginate-TMC-mPEG capsules allows cell growth, proliferation and long-term survival of stem cells (Hashemi and Kalalinia 2015). Furthermore, according to Fig. 2, the viable cells were constant on days 5 and 7 after encapsulation. Low space for the proliferation of cells may lead to decreasing in cell growth rate. Thus, the 5th day after microencapsulation may be the optimal time for cell differentiation induction in future works.

To investigate three-layered microcapsules in mESCs immunocamouflaging, specifically, IL-2 secretion against the encapsulated mESCs in alginate, alginate-TMC and alginate-TMC-mPEG microcapsules was measured. IL-2 secretion increasing over time showed that lymphocytes recognized the mESCs during the incubation time. Nevertheless, encapsulation of mESCs prevented direct contact between mESCs and lymphocytes, and as a result it hindered the immune system stimulation. Here, we have demonstrated that mPEG attachment to the surface of alginate-TMC microcapsules decreased the immunological response. Therefore, recognition of the mESCs and IL-2 secretion was reduced.

In cell encapsulation, the cells are immobilized inside a semi-permeable matrix to protect the cells. Other potential advantages of this method may include cell expansion and self-renewal potency or direct cell differentiation toward a desired lineage. Consequently, cell encapsulation 


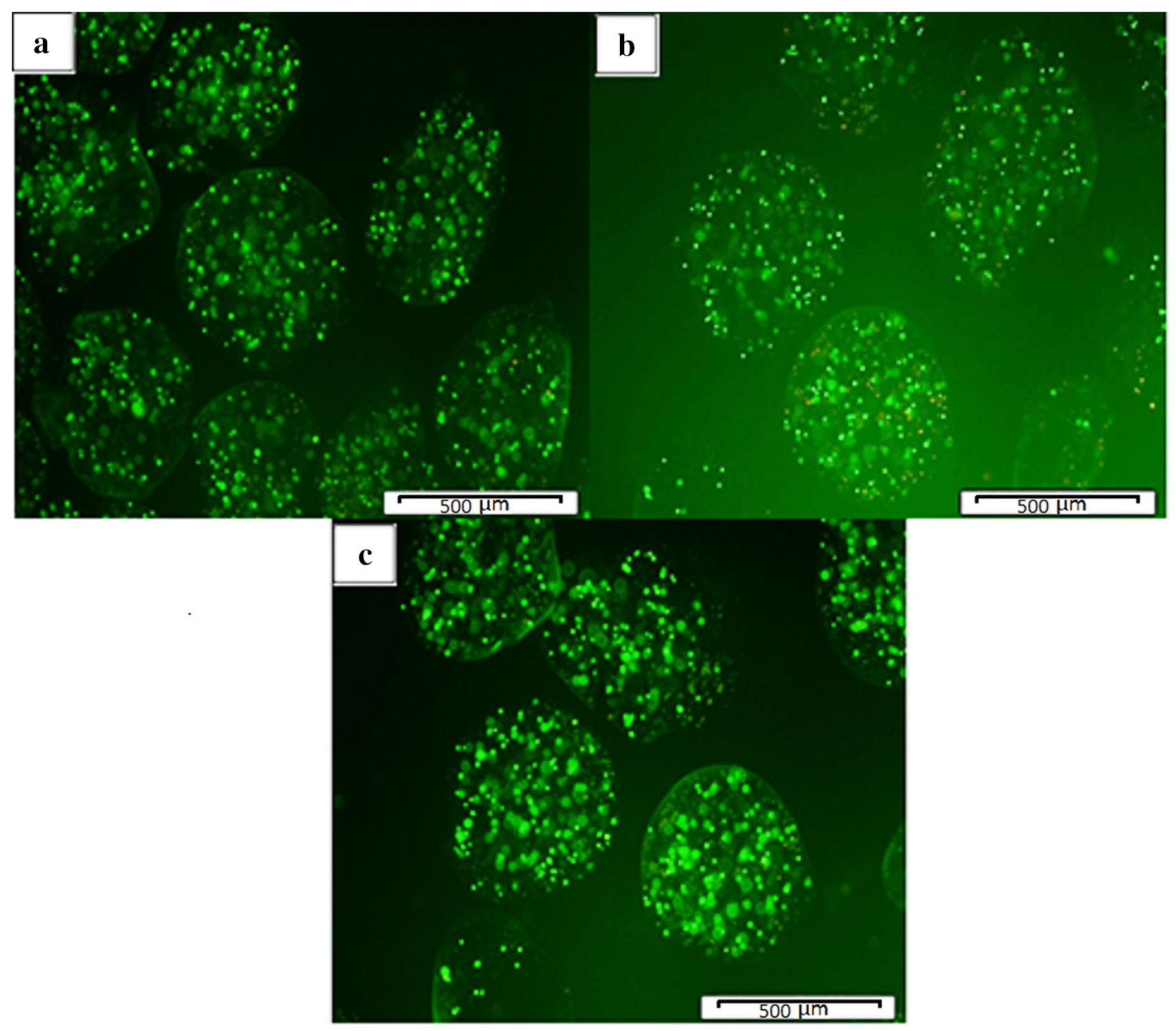

Fig. 4 Viability of the encapsulated mESCs in alginate (a), alginate-TMC (b), alginate-TMC-PEG (c) microcapsules, after co-culturing with lymphocytes; using AO/PI assay (scale bar $=500 \mu \mathrm{m})$

is considered as an attractive method for many clinical applications.

\section{Conclusion}

mESCs were encapsulated in alginate, alginate-TMC and alginate-TMC-mPEG microcapsules, separately. A three-layer encapsulation of mESCs prevented direct contact between mESCs and lymphocytes and it hindered their recognition by the immune system. Subsequently, the capsules could adequately protect the cells against the co-cultured lymphocytes at this stage. It also allowed the cell growth and proliferation. In our previous work, we introduced encapsulation and PEGylation system for islets transplantation simultaneously. Here, we proposed it for stem cell capsulation for future works that stem cells should be differentiated to a specific cell line such as bone or insulin-producing cells, inside the capsules and then transplanted.

Funding This study was supported by Tarbiat Modares University.

\section{Compliance with Ethical Standards}

Conflict of Interest The authors declare that they have no conflict of interest.

Ethical approval All applicable international, national, and/or institutional guidelines for the care and use of animals were followed.

Human participants This article does not contain any studies with human participants performed by any of the authors. 
Open Access This article is distributed under the terms of the Creative Commons Attribution 4.0 International License (http://creativeco mmons.org/licenses/by/4.0/), which permits unrestricted use, distribution, and reproduction in any medium, provided you give appropriate credit to the original author(s) and the source, provide a link to the Creative Commons license, and indicate if changes were made.

\section{References}

Al-Rammah TY (2014) Alginate microencapsulation of stem cells as alternative source to the limited supply of donor tissue. Biomed Research 25:276-280

Azadi SA, Vasheghani-Farahani E, Hashemi-Najafabadi S, Godini A (2016) Co-encapsulation of pancreatic islets and pentoxifylline in alginate-based microcapsules with enhanced immunosuppressive effects. Prog Biomater 5:101-109. https://doi.org/10.1007/s4020 4-016-0049-3

Chayosumrit M, Tuch B, Sidhu K (2010) Alginate microcapsule for propagation and directed differentiation of hESCs to definitive endoderm. Biomaterials 31:505-514. https://doi.org/10.1016/j. biomaterials.2009.09.071

Coates J (2000) Interpretation of infrared spectra a practical approach. In: Meyers RA (ed) Encyclopedia Anal Chem. Wiley, Chichester, pp 10815-10837. https://doi.org/10.1002/9780470027318

Hashemi M, Kalalinia F (2015) Application of encapsulation technology in stem cell therapy. Life Sci 143:139-146. https://doi. org/10.1016/j.lfs.2015.11.007

Hernández RM, Orive G, Murua A, Pedraz JL (2010) Microcapsules and microcarriers for in situ cell delivery. Adv Drug Deliv Rev 62:711-730. https://doi.org/10.1016/j.addr.2010.02.004

Hu X, Zhang C, Chen W, Wang Z, Zhang X (2013) Synthesis and characterization of low-toxicity $\mathrm{N}$-caprinoyl-N-trimethyl chitosan as self- assembled micelles carriers for osthole. Int J Nanomedicine 8:3543-3558. https://doi.org/10.2147/IJN.S46369

Kim J, Sachdev P, Sidhu K (2013) Sciencedirect alginate microcapsule as a 3D platform for the efficient differentiation of human embryonic stem cells to dopamine neurons. Stem Cell Research 11:978-989. https://doi.org/10.1016/j.scr.2013.06.005
Moshaverinia A, Xu X, Chen C, Akiyama K, Snead ML, Shi S (2013) Dental mesenchymal stem cells encapsulated in an alginate hydrogel co-delivery microencapsulation system for cartilage regeneration. Acta Biomater 9:9343-9350. https://doi.org/10.1016/j.actbi o.2013.07.023

Mourya VK, Inamdar NN (2009) Trimethyl chitosan and its applications in drug delivery. J Mater Sci-Mater Med 20:1057-1079. https://doi.org/10.1007/s10856-008-3659-z

Nabavimanesh MM, Hashemi-Najafabadi S, Vasheghani-Farahani E (2015) Islets immunoisolation using encapsulation and PEGylation, simultaneously, as a novel design. J Biosci Bioeng 119:486491. https://doi.org/10.1016/j.jbiosc.2014.09.023

Paredes JuÃ $i r e z$ GA, Spasojevic M, Faas MM, de Vos P (2014) Immunological and technical considerations in application of alginatebased microencapsulation systems. Front Bioeng Biotechnol 2:1-15. https://doi.org/10.3389/fbioe.2014.00026

Pavia D, Lampman G, Kriz G, Vyvyan J (2008) Introduction to spectroscopy. Cengage Learning, Boston

Sadeghi AM, Erfan M, Amini M (2004) Synthesis and characterization of $N$-diethymethyl chitosan. Polymer J 13:431-436

Sun AM (1988) Microencapsulation of pancreatic islet cells: a bioartificial endocrine pancreas. Meth Enzymol 137:575-580

Tam SK, Bilodeau S, Dusseault J, Langlois G, Hallé JP, Yahia LH (2011) Biocompatibility and physicochemical characteristics of alginate-polycation microcapsules. Acta Biomater 7:1683-1692. https://doi.org/10.1016/j.actbio.2010.12.006

Trif M, Ansorge-Schumacher M, Socaciu C (2007) Application of FTIR spectroscopy for determination of oxidation of encapsulated sea buckthorn oil. XVth int workshop on bioencapsulation, Vienna, Au. Sept 6-8

Wang N, Adams G, Buttery L, Falcone FH, Stolnik S (2009) Alginate encapsulation technology supports embryonic stem cells differentiation into insulin-producing cells. J Biotechnol 144:304-312. https://doi.org/10.1016/j.jbiotec.2009.08.008

Publisher's Note Springer Nature remains neutral with regard to urisdictional claims in published maps and institutional affiliations. 